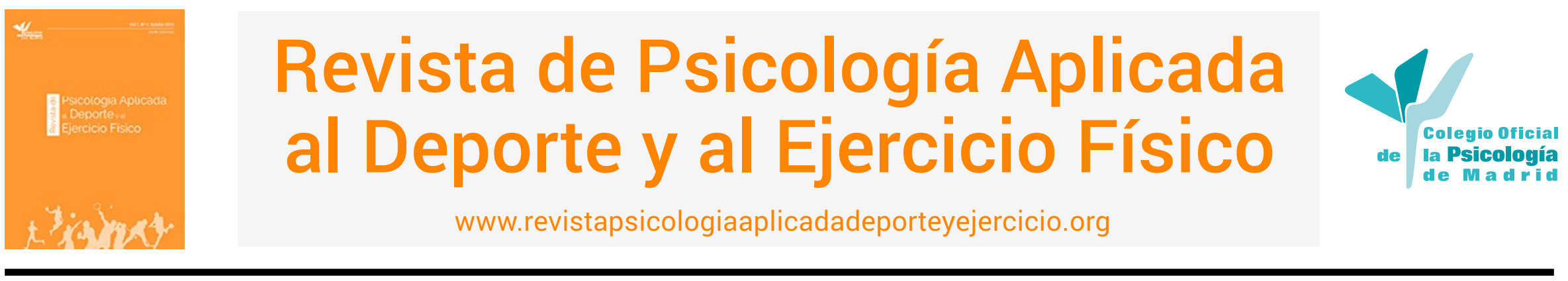

\title{
Preparación psicológica para los Juegos Paralímpicos y afrontamiento de lesión: un caso en taekwondo
}

\author{
Belén Olmedilla-Caballero', Isabel María Moreno-Fernández², Verónica Gómez-Espejo', Aurelio Olmedilla-Zafra' \\ ${ }^{1}$ Universidad de Murcia, España, ${ }^{2}$ Universidad Autónoma de Barcelona, España
}

RESUMEN: Se presenta un estudio de caso de un deportista (parataekwondo, CAR Infanta Cristina, Murcia) de 25 años. La demanda inicial del deportista es gestionar adecuadamente pensamientos y creencias que le afectan al rendimiento como: "Al entrar a competir siento que no me sale, que me pongo nervioso, sin embargo estoy muy bien en los entrenos". Al iniciar el trabajo psicológico con el deportista en la entrevista indica que tiene una lesión que viene arrastrando hace tiempo, pero que tras valoración médica, y junto a su entrenador toman la decisión de una rehabilitación conservadora para intentar llegar a los Juegos Paralímpicos, tras los que se operaría. La intervención psicológica, combinando un trabajo específico para la lesión y uno más general para la competición, se centra en fortalecer la confianza en los combates, incrementar la capacidad de concentración en competición, controlar el dolor y fortalecer la adherencia al programa de rehabilitación.

PALABRAS CLAVES: Lesión, intervención psicológica, Juegos paralímpicos, taekwondo.

\section{Psychological preparation for the Paralympic Games and coping with injury: a case report in taekwondo}

ABSTRACT: The case of a 25-year-old male athlete (parataekwondo, CAR Infanta Cristina, Murcia) is reported. The initial demand of the athlete was to be able to adequately manage the thoughts and beliefs affecting his performance such as: "When I am about to compete, I feel as if I could perform, I get nervous; however, I feel very good while training." At the start of psychological work with the athlete, he reported during the interview that he had been dragging an injury for a long time, but after medical evaluation, he and his coach decided on a conservative rehabilitation to try and reach the Paralympic Games, after which he would undergo surgery. The psychological intervention, combining specific work for the injury and more general work for competition, focuses on strengthening confidence for fighting, increasing concentration in competition, pain control, and strengthening adherence to the rehabilitation program.

KEYWORDS: Injuries, psychological intervention, Paralympics Games, taekwondo.

\section{Preparação psicológica para os Jogos Paraolímpicos e adaptação a lesão: um caso no taekwondo}

RESUMO: Apresenta-se um estudo de caso de um atleta de 25 anos (parataekwondo, CAR Infanta Cristina, Múrcia). A exigência inicial do atleta é gerir adequadamente pensamentos e crenças que afetam o seu desempenho, como: "Ao entrar para competir, sinto que não me corre bem, fico nervoso, ainda que seja muito bom nos treinos". Ao iniciar o trabalho psicológico com o atleta, na entrevista, ele indica que tem uma lesão que o acompanha há muito tempo. Mas que, após avaliação médica e juntamente com o

Agradecimientos/Financiación: Este estudio se ha realizado dentro del Contrato/Programa entre el Centro de Tecnificación Deportiva (CTD) de la Comunidad Autónoma de la Región de Murcia y la Universidad de Murcia (UMU31047-GINVEST10294).

Isabel María Moreno-Fernández es psicóloga en la Universidad Autónoma de Barcelona.

Verónica Gómez-Espejo (iD) https:// orcid.org/0000-0003-4892-7047 es psicóloga en la Universidad de Murcia.

Belén Olmedilla-Caballero (iD https:// orcid.org/0000-0002-8309-9442 es psicóloga en la Universidad de Murcia.

Aurelio Olmedilla Zafra (D) https:// orcid.org/0000-0002-2389-0515 es psicólogo en la Universidad de Murcia.

La correspondencia sobre este artículo debe enviarse al último autor a Calle Almagro, 22, 30 1zda., 30530, Cieza, Murcia (España). Mail: olmedilla@um.es 
seu treinador, foi tomada a decisão de uma reabilitação conservadora para tentar alcançar os Jogos Paraolímpicos e que, depois disso, seria operado. A intervenção psicológica, combinando um trabalho específico para a lesão e um mais geral para a competição, concentra-se no fortalecimento da confiança nos combates, no aumento da capacidade de concentração na competição, no controlo da dor e no fortalecimento da adesão ao programa de reabilitação.

PALAVRAS-CHAVE: lesão, intervenção psicológica, jogos paraolímpicos, taekwondo.

Artículo recibido: 13/04/2020 | Artículo aceptado: 31/05/2020

La investigación y la intervención psicológica en deportistas se puede encontrar en muchas esferas y ámbitos, como es el caso de los deportes paralímpicos, de los que han sido publicados algunos trabajos de gran interés. Se pueden encontrar estudios muy recientes en los que se analizan diferentes aspectos psicológicos y psicosociales de la práctica de deporte paralímpico (Alexander et al., 2020; Martin et al., 2019; Puce et al., 2017; Torralba et al., 2017), incluso propuestas, muy interesantes, de gran calado social, partiendo de una conceptualización del activismo paralímpico en el contexto de cambio de comportamiento desde una perspectiva cualitativa (Haslett et al., 2020), en la que sobresale el valor social, la política de identidad y el desafío ante la dicotomía capacidad-discapacidad.

Por otro lado, también se pueden encontrar algunos estudios que presentan programas de intervención psicológica con deportistas paralímpicos, como la aplicación del Mindfulness para la reducción del estrés de líderes paralímpicos (Lundqvist et al., 2018), los efectos del entrenamiento de habilidades psicológicas para tenistas paralímpicos coreanos (Lim et al., 2018), o aplicaciones integrales para deportistas, entrenadores y técnicos dentro del contexto de unos Juegos Paralímpicos (Aragón, 2002).

En general, la importancia de los factores psicológicos en el rendimiento deportivo ha sido ampliamente estudiada (Abdullah et al., 2016; Álvarez et al., 2014; Carazo y Araya, 2010; Gross et al., 2018; Kristiansen et al., 2008; MacNamara et al., 2010; Olmedilla et al., 2017; Robles-Rodríguez et al., 2020), demostrándose que aunque los factores psicológicos en sí mismos no producen un aumento del rendimiento, sí promueven que el deportista alcance su potencial máximo cuando median con sus conocimientos técnicos, tácticos y físicos (Mahamud et al., 2005; Sotoodeh et al., 2012). Por tanto, el entrenamiento psicológico debería estar instaurado dentro de la metodología de trabajo del equipo o el deportista, trabajándose en los entrenamientos como se trabajan aspectos tácticos o técnicos, para posibilitar este rendimiento óptimo del deportista.

En relación a esto, son numerosos los estudios en los que se llevan a cabo intervenciones psicológicas en depor- tistas o en equipos buscando esta mejora del rendimiento (Golby y Wood, 2016; Moreno-Fernández et al., 2019; Olmedilla et al., 2019), integrando, cada vez más, este trabajo psicológico en el entrenamiento del deportista, aunque aún queda mucho por hacer en este aspecto. Además, algunos autores sugieren la utilización de diseños de investigación de caso único para comprender mejor como funciona una intervención psicológica profesional (Kinugasa, 2013), encontrando en estos últimos años algunos trabajos muy enriquecedores (Abenza et al., 2014; Ashbrook et al., 2018; Palmi et al., 2018; Peris-Delcampo et al., 2019; Smith et al., 2019; Turner et al., 2020).

Un tema que ha suscitado gran interés dentro de la investigación aplicada ha sido cómo se relacionan las variables psicológicas asociadas al rendimiento con las lesiones deportivas. El Modelo Global Psicológico de las Lesiones Deportivas (MGPSLD, Olmedilla y García-Mas, 2009) considera la intervención psicológica como una de las principales líneas de investigación en la relación psicología y lesión deportiva, considerando el abordaje multidisciplinar como básico, y estableciendo las variables psicológicas (estrés, recursos de afrontamiento, adherencia a la rehabilitación) y situacionales (tipo de deporte, competiciones versus entrenamientos) más relevantes dentro de los tres ejes (causal, temporal y conceptual) que lo componen. Uno de los desarrollos del MGPSLD es el llevado a cabo por Liberal et al. (2014), centrando el énfasis en el impacto emocional de la lesión sobre el deportista desde una perspectiva que incluye el bienestar psicológico percibido. En este sentido, aquellos deportistas experimentados (con cierta historia de lesiones) manifestaron mayor percepción de control sobre su situación de lesión mostrando buenos niveles de bienestar psicológico, haciendo un continuum entre su vida deportiva y su vida personal. Por otro lado, la lesión puede tener un impacto importante sobre el estado de ánimo y los procesos emocionales del deportista incidiendo directamente en las conductas de adherencia a la rehabilitación (Abenza, Olmedilla y Ortega, 2010).

Los deportes de combate son deportes de alto riesgo, peligrosos para la salud física más que otros deportes, 
debido al alto contacto que hay entre los deportistas, aunque cuando el deportista se inicia a edad temprana se produce una adaptación que, de alguna manera, supone un factor de protección ante la lesión (Martín et al., 2018). Las lesiones más frecuentes en estos deportes son de carácter leve, como hematomas y laceraciones (Martín et al., 2018; McPherson y Pickett, 2010; Wilkerson, 1997).

Teniendo en cuenta todo lo anterior, el objetivo de este artículo es presentar el trabajo psicológico realizado con un deportista de alto rendimiento de cara a su preparación para los Juegos Paralímpicos de Tokyo 2020 (suspendido, COVID-19), y sometido a la incertidumbre de realizar su preparación arrastrando una lesión.

\section{Descripción del caso}

Varón deportista de 25 años que compagina su práctica deportiva con estudios de ingeniería en la universidad. Su especialidad es el Taekwondo, concretamente para-taekwondo, con un amplio historial deportivo, con más de 15 años de experiencia competitiva. Destaca el proclamarse Campeón del Mundo en los años 2009, 2011 y 2013, además de Campeón de Europa en 2012 y 2014. El deportista se encontraba, en el momento del estudio, en el Centro de Tecnificación Deportiva (CAR) "Infanta Cristina" de Los Alcázares, Murcia, España, donde entrenaba 6 días a la semana, con unas 25 horas de entrenamiento semanal aproximadamente. El CAR es un centro ideal para el entrenamiento de alto nivel, dando cabida a deportistas de diferentes disciplinas deportivas, además de acoger concentraciones tanto permanentes como temporales de diferentes federaciones, clubes o equipos, tanto en periodos de pretemporada, como en cualquier momento de la competición. El CAR tiene un Servicio de Psicología del Deporte dirigido por un psicólogo deportivo con amplio historial profesional y miembro de la División de Psicología de la Actividad Física y del Deporte (PACFD) del Colegio Oficial de Psicólogos, que es quien atendió al deportista.

\section{Demanda psicológica inicial}

El deportista llega a la consulta de psicología con un objetivo muy claro: mejorar su preparación de cara a los juegos paralímpicos de Tokio 2020. Durante la entrevista, con la que se iniciaba el trabajo psicológico con el deportista, éste nos indica que presenta una lesión, tratándose de una lesión grave en deportistas de élite (rotura total del ligamento cruzado anterior de la rodilla izquierda). Además, explica que después del primer impacto y tras la valoración médica, junto a su entrenador toman la decisión de una rehabilitación conservadora para intentar llegar a los juegos paraolímpicos, tras los que se operaría.

\section{Intervención psicológica}

Se presenta el proceso de intervención psicológica realizado, indicando los aspectos relevantes de la evaluación, los objetivos del trabajo psicológico y la inserción de éste dentro del programa de trabajo con el deportista.

\section{Evaluación de los aspectos relativos a la lesión}

Análisis de los antecedentes de la lesión, expectativas y estado psicológico. El deportista, con un estado de ánimo muy motivado, se encontraba en situación de pretemporada (octubre 2018) por lo que se dedicaba a la planificación deportiva del año (Mundial en febrero de 2019; Campeonato de Europa, abril 2019; Open de Asia, septiembre 2019). Sus expectativas deportivas eran en primer lugar, lograr la clasificación para los Juegos Paralímpicos de Tokio 2020), ganar el mundial, y, por último, conseguir la mayoría de puntos en el ranking. Respecto a las competiciones realizadas antes de la lesión, se encuentran el Mundial, el Open de África, USA y México, y los Continentales Americano, asiático, europeo y Oceanía.

Valoración de las consecuencias objetivas y subjetivas de la lesión. Respecto a las consecuencias objetivas, el deportista explicó que la lesión supuso un corte para la preparación de los campeonatos. Por otro lado, y atendiendo a las consecuencias subjetivas, el deportista explicó que lo que más le preocupaba era el pensar que no podría clasificarse para los JJOO.

Valoración del proceso de rehabilitación de la lesión y su impacto psicológico, emocional y deportivo. Lo primero que se llevó a cabo fueron las pruebas diagnósticas y la búsqueda de soluciones. El primero en intervenir fue el médico con una resonancia, en la que se veía una posible rotura parcial del Ligamento, por lo que había que contrastar con el traumatólogo. Este profesional le indica que no parece estar roto pero que se necesita realizar un seguimiento y valoración. De esta manera, intervino el fisioterapeuta, que en un primer momento iba a llevar a 
cabo 10 sesiones, pero finalmente fueron 20 (1 día/semana), tras las recomendaciones del traumatólogo. Durante estas 10 primeras sesiones el deportista se encontraba asustado, sin embargo, con confianza en la recuperación. En las otras 10 sesiones restantes, comienza a desconfiar y sentirse abatido ya que sus expectativas habían cambiado (no creía que pudiera llegar a competir a alto nivel). Mientras se llevaban a cabo las intervenciones con el fisioterapeuta, el deportista junto al entrenador decide la continuación del entrenamiento y competición para no perder la ocasión de ir a Tokio. Tras esta decisión, el deportista fue a probar en competición con bajas expectativas, únicamente con la idea de sacar los máximos puntos posibles. Finalmente, el deportista se encontraba ansioso puesto que visita a los médicos del Comité Olímpico Español (COE), los cuales confirman la rotura del ligamento y la necesidad de operación. No obstante, el deportista, con tranquilidad, se centró en buscar soluciones para que no se anularan la actividad deportiva.

Valoración de la vuelta a la actividad deportiva, y la decisión de seguir entrenando y compitiendo. La actividad deportiva fue adaptada para que el deportista pudiera entrenar sin necesidad de forzar demasiado. Además, éste debía de llevar a cabo sesiones con el fisioterapeuta para realizar trabajo de descarga, y un seguimiento de la lesión con los médicos del COE. Finalmente, el deportista decidió no operarse, debido a que la rodilla le era funcional y el dolor no iba a más, para intentar clasificarse lesionado y dejar la operación para un momento posterior, para no perder la temporada entera. A partir de esa decisión, compitió en el Mundial, Open de África, de USA y de México, no cumpliendo con las expectativas en ninguna de estas competiciones.

\section{Objetivos del trabajo psicológico específico en relación a la lesión}

Tras entrevista con el deportista, y de mutuo acuerdo, se acordó plantear la consecución de los siguientes objetivos:

1. Fortalecer la confianza en los combates

2. Incrementar la capacidad de concentración en competición

3. Controlar el dolor

4. Fortalecer la adherencia al programa de rehabilitación Para la consecución de estos objetivos se planteó una intervención de 6 meses, hasta junio de 2019, justo antes del parón vacacional y el Open de Asia (septiembre, 2019). Se realizaron 14 sesiones presenciales, de una hora de duración, en el CAR y con una frecuencia aproximada de 15 días, aunque estaba en función del desarrollo de la intervención.

Para la consecución de los objetivos 1 y 2 se implementaron diferentes técnicas, como establecimiento de objetivos (Olmedilla, et al., 2018; Weinberg y Gould, 2018), plan psicológico de competición (Abenza et al., 2017; González-Hernández, 2017; Pedraza-Ramírez, 2019) y análisis cognitivo de competiciones (Vallarino et al., 2020; Zamora et al., 2017). Para el objetivo 3, control del dolor, se hizo una intervención basada en la terapia de desensibilización y reprocesamiento mediante movimientos oculares (EMDR) de Shapiro (1995) y Shapiro y Forrest (2001), junto a entrenamiento en práctica imaginada (García-Naveira, 2017;

Tabla 1. Trabajo psicológico específico en los momentos del proceso de rehabilitación

\begin{tabular}{clll}
\hline Mo & Competición/entrenos & \multicolumn{1}{c}{ Percepción dolor/molestias } & Trabajo psicológico \\
\hline 1 & $\begin{array}{l}3 \text { competiciones + entre- } \\
\text { namientos. }\end{array}$ & $\begin{array}{l}\text { Primera competición lesionado, bastante molesto. } \\
\text { Entrenamientos preparatorios para los campeona- } \\
\text { tos. }\end{array}$ & Pautas de afrontamiento + EMDR. \\
\hline $\begin{array}{l}\text { Visita al médico del COE } \\
\text { y entrenos de cara a los } \\
\text { próximos campeonatos. }\end{array}$ & $\begin{array}{l}\text { Se confirma la lesión que ya sabíamos que tenía- } \\
\text { mos. Decidimos no operar. }\end{array}$ & $\begin{array}{l}\text { Práctica imaginada: control del dolor y búsqueda } \\
\text { de calma, de relajación. }\end{array}$ \\
\hline & $\begin{array}{l}\text { Periodo de entrenos + } \\
\text { competiciones. }\end{array}$ & $\begin{array}{l}\text { Un poco de molestias más intensas en la parte de } \\
\text { la temporada en las que los entrenamientos fueron } \\
\text { más de carga. }\end{array}$ & $\begin{array}{l}\text { Práctica imaginada. Preparación planes psicológi- } \\
\text { cos para las próximas competiciones. }\end{array}$ \\
\hline $\begin{array}{l}\text { Periodo de exámenes }+ \\
\text { entrenos + competición. }\end{array}$ & $\begin{array}{l}\text { Mucha molestia, debido al estrés y la carga de es- } \\
\text { tos meses. Además, la temporada se está hacien- } \\
\text { do larga. Tengo una recaída de la lesión. }\end{array}$ & $\begin{array}{l}\text { Registros percepción de dolor de la rodilla. } \\
\text { Nos centramos más en el control del dolor. } \\
\text { EMDR. }\end{array}$ \\
\hline
\end{tabular}


Olmedilla et al., 2010) y evaluación del dolor mediante la escala EVA (Chica et al., 2019; Gil-Moreno et al., 2017). Para la consecución del 4 objetivo, fortalecer la adherencia al programa de rehabilitación, se utilizó el establecimiento de objetivos y el trabajo conjunto con el fisioterapeuta (Abenza, Olmedilla, Ortega, et al., 2010; Abenza et al., 2011).

\section{Inserción del trabajo psicológico específico para la lesión dentro del programa de entrenamiento}

La inserción del trabajo psicológico específico para la lesión se explica y detalla en la Tabla 1.
En la Tabla 2 se recogen cuatro de los aspectos psicológicos en los que se prestó especial atención, el estado de ánimo, la disposición psicológica, las expectativas y el apoyo social percibido, en los diferentes momentos del proceso de rehabilitación.

Como ya se ha comentado más arriba, para la consecución de cada objetivo se llevaron a cabo diferentes técnicas y estrategias psicológicas. A modo de ejemplo, se indican algunas de las utilizadas en este trabajo. En la Tabla 3 se puede ver un ejemplo, relacionado con los objetivos 1 y 2 , donde se presenta la utilización de un Plan de Competición.

Igualmente, para la consecución del objetivo 3, entre

\section{Tabla 2. Trabajo psicológico específico en el proceso de rehabilitación}

\begin{tabular}{|c|c|c|c|c|c|}
\hline Mo & Rehabilitación & Estado de ánimo & Disposición psicológica & Expectativas & Apoyo social \\
\hline 1 & $\begin{array}{l}\text { Rehabilitación. Propio- } \\
\text { cepción y ejercicios } \\
\text { específicos para el } \\
\text { fortalecimiento de la } \\
\text { zona. }\end{array}$ & $\begin{array}{l}\text { Al principio un poco de } \\
\text { incertidumbre porque no } \\
\text { sabía cómo reaccionaría } \\
\text { la rodilla. } \\
\text { Luego fue un mes muy } \\
\text { bueno sacando bastantes } \\
\text { puntos para el ranking. }\end{array}$ & $\begin{array}{l}\text { Buena disposición. } \\
\text { Gracias al trabajo psico- } \\
\text { lógico creo que a estas } \\
\text { alturas estamos en la } \\
\text { posición que estamos. }\end{array}$ & $\begin{array}{l}\text { Seguir preparando y } \\
\text { mejorando aspectos } \\
\text { psicológicos para las } \\
\text { siguientes competicio- } \\
\text { nes y la lesión. }\end{array}$ & $\begin{array}{l}\text { La verdad que mi familia } \\
\text { y amigos siempre han } \\
\text { estado ahí poyándome } \\
\text { todos los días y me siento } \\
\text { muy respaldado. } \\
\text { Mi entrenador también } \\
\text { está muy involucrado y } \\
\text { eso ayuda a que tu estés } \\
\text { metido 100\%. } \\
\text { A parte el psicólogo no } \\
\text { me deja respirar y está } \\
\text { muy involucrado también. }\end{array}$ \\
\hline 3 & $\begin{array}{l}\text { Mismo que en el apar- } \\
\text { tado del momento } 2 \text {. }\end{array}$ & $\begin{array}{l}\text { Mismo que en el apartado } \\
\text { del momento } 2 \text {. }\end{array}$ & $\begin{array}{l}\text { Mismo que en el apar- } \\
\text { tado del momento } 2+ \\
\text { preparar las competicio- } \\
\text { nes ajustando aspectos } \\
\text { tácticos que nos habían } \\
\text { servido. }\end{array}$ & $\begin{array}{l}\text { Mismo que en el aparta- } \\
\text { do del momento } 2 \text {. }\end{array}$ & $\begin{array}{l}\text { Mismo que en el apartado } \\
\text { del momento } 2 \text {. }\end{array}$ \\
\hline
\end{tabular}


Tabla 3. Ejemplo de plan psicológico de competición utilizado

Indicar la estrategia (táctica) a seguir en la competición ¿cómo he preparado el combate? ¿Cómo voy a realizar el combate? ¿Cómo quiero competir?

Combate de distancia larga, no dejando que se meta en el cuerpo a cuerpo el contrincante y de tener la anticipación siempre preparada.

¿Qué posibles dificultades puedo encontrarme en el desarrollo del combate?

1. Distancias cortas
2. No llevar yo el control del combate

Para cada una de estas dificultades ¿Qué plan alternativo tengo preparado?

1. No favorecer intercambios con bloqueos fuertes en el cuerpo a cuerpo

2. Ir a remolque en el combate

Valoración general del desarrollo del combate

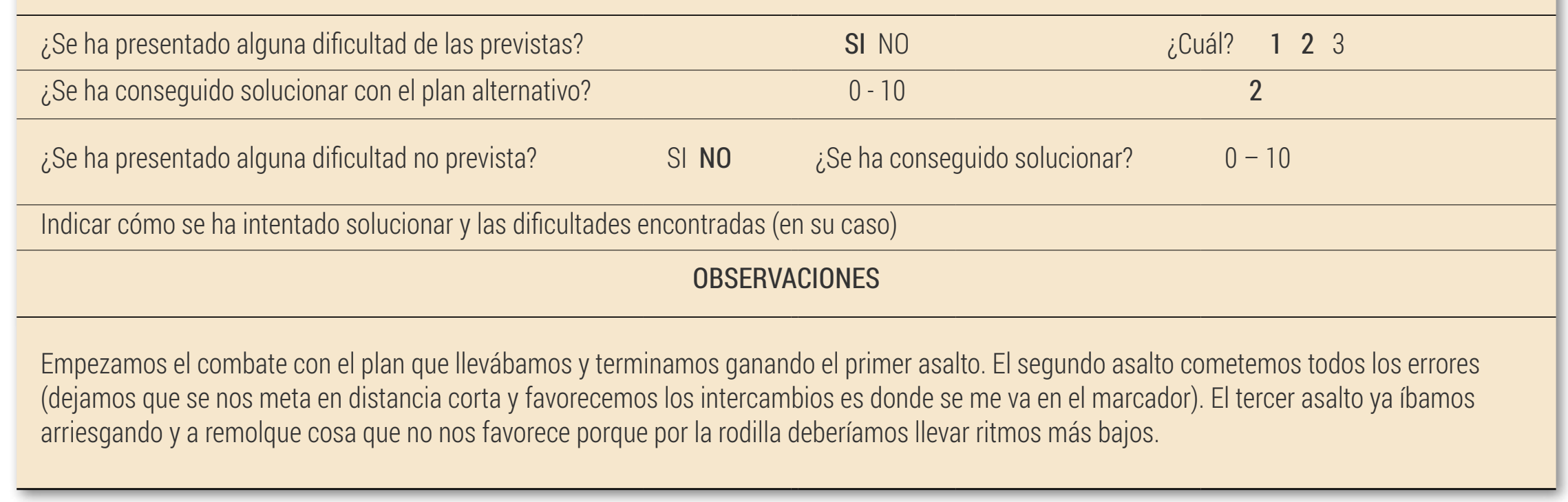

otros, se utilizó un protocolo de evaluación del dolor para 4 momentos del día, justo al levantarse, justo antes de comer, a las 18.00 horas y justo antes de acostarse (ver

\section{Figura 1. Evaluación del dolor}

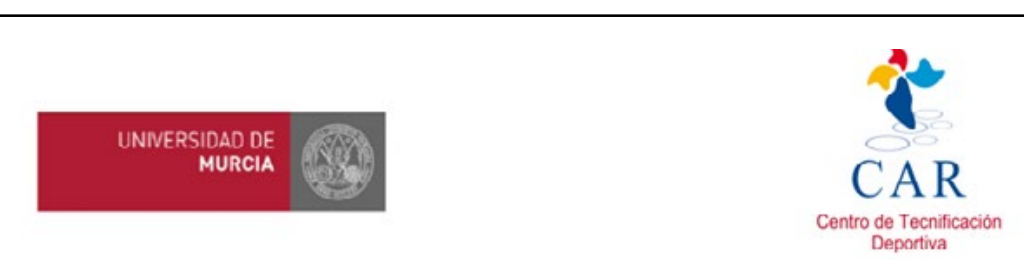

ESCALA EVA - EVALUACIÓN DEL DOLOR

Más abajo hay una imagen que representa en una escala de 0 a 10 la intensidad del dolor que usted siente Por favor, cumplimente sus datos personales, la fecha del dia en que realiza la evaluación del dolor y cumplimente, para cada dia, 4 veces la Escala del Dolor. Debe de rodear con un círculo o señalar con una cumplimente, para cada dia, 4 veces la Escala del Dolor. Debe de rodear con un circulo o señala
cruz $(\mathrm{X})$ donde crea que la Escala representa el dolor que usted siente en ese mismo momento.

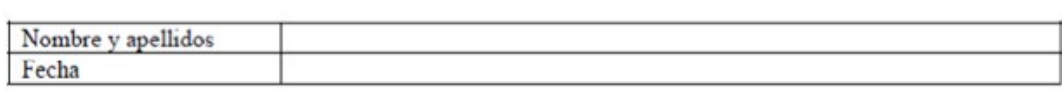

Fech

\begin{tabular}{|l|l|}
\hline Registro 1 & Por la mañana, justo al levantarse \\
\hline
\end{tabular}

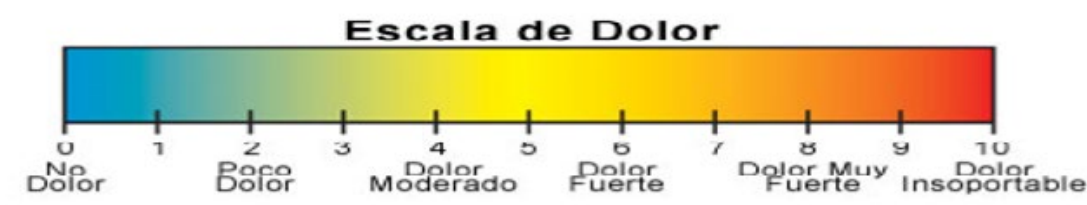

Figura 1), y el trabajo específico en EMDR (auditivo, 30 minutos diarios, auto-administrado), que era registrado por el deportista en cada sesión dolor (ver Figura 2).

\section{Figura 2: Registro de sesión EMDR}

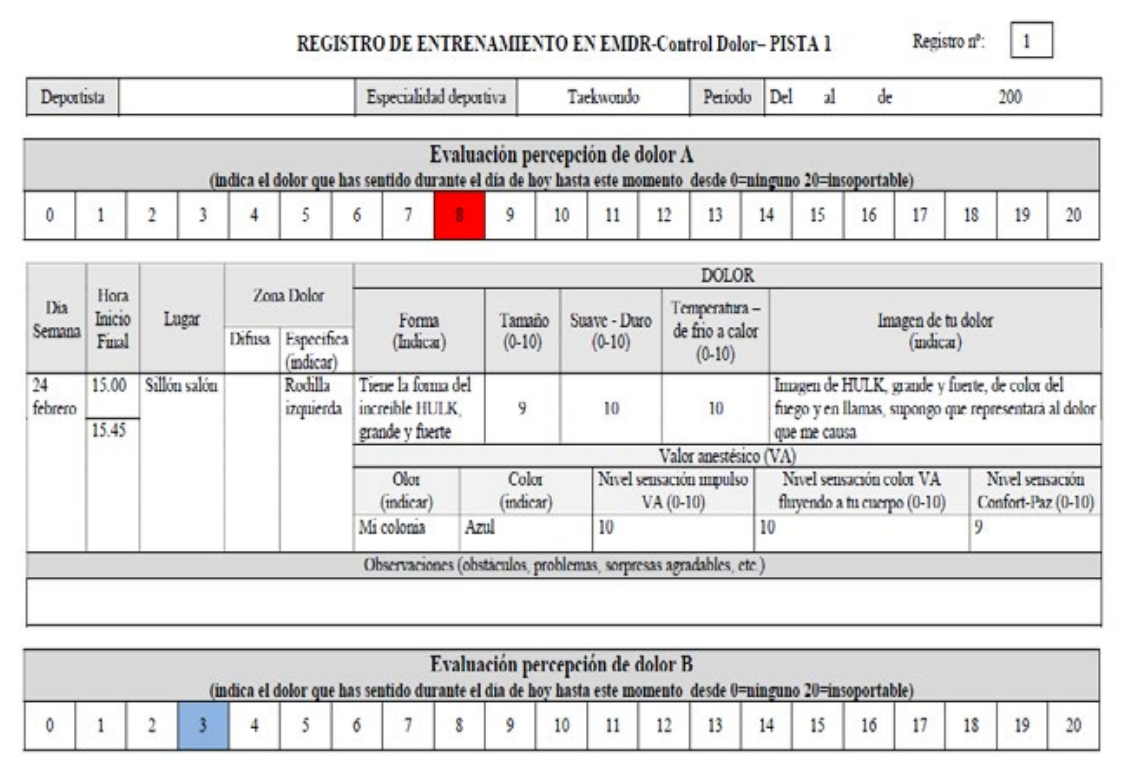


Tabla 4. Registro del fisioterapeuta sobre la adherencia al programa de rehabilitación

\begin{tabular}{|c|c|c|c|c|c|}
\hline 1. Asistencia. & \multicolumn{2}{|c|}{ sí } & \multicolumn{3}{|c|}{ NO } \\
\hline 2. Puntualidad. & \multicolumn{2}{|c|}{ sí } & \multicolumn{3}{|c|}{ NO } \\
\hline PERCEPCIÓN DEL PROFESIONAL SANITARIO & Mín & & & & MÁX \\
\hline $\begin{array}{l}\text { 3. Grado de cumplimiento de las tareas prescritas, instrucciones o consejos } \\
\text { médicos. }\end{array}$ & 0 & 1 & 2 & 3 & 4 \\
\hline 4. Grado de esfuerzo físico en los ejercicios de rehabilitación. & 0 & 1 & 2 & 3 & 4 \\
\hline 5. Grado de dominio de las técnicas o ejercicios de rehabilitación. & 0 & 1 & 2 & 3 & 4 \\
\hline 6. Grado de receptividad del deportista ante los cambios en el tratamiento de rehabilitación. & 0 & 1 & 2 & 3 & 4 \\
\hline
\end{tabular}

Tabla 5. Valoración por parte del deportista del trabajo psicológico realizado

¿Estás satisfecho/a con la preparación psicológica realizada? (indica de $0=$ nada satisfecho, a 10=muy satisfecho; y responde las 2 siguientes cuestiones)

Indica los aspectos que más te han gustado y razona porqué

Tener objetivos claros, obtener información relevante a la hora de mejorar aspectos (antes, durante y después) de una competición, buscar el mejor enfoque ante los problemas que te surgen en el momento.

Indica los aspectos que menos te han gustado y razona porqué

La parte de la visualización creo que es la más compleja que he trabajado a nivel psicológico porque no podía sacarle partido en la competición.

¿En qué medida crees que te ha servido para tu preparación deportiva general?

Indica los aspectos que más te han gustado y razona porqué

Mayor control del estrés en competición, gestionar situaciones de una tensión alta durante la preparación y el desarrollo de una competición, controlar la ansiedad que genera una lesión o la bajada de peso y mejorar tácticamente en los aspectos ofensivos, pero más en lo defensivos.

Indica los aspectos que menos te han gustado y razona porqué

No tenía una base psicológica así que creo que todo me ha ayudado

¿En qué medida consideras que te ha ayudado para afrontar la lesión y continuar con tu preparación en entrenamientos y competiciones?

Indica los aspectos que más te han servido y razona porqué

La gestión del dolor y ayudarme a planificarme mejor han sido vitales porque tenía una rutina clara y trabajaba englobando la lesión dentro del plan competición. A parte de las sesiones de fisioterapia y el seguimiento que hemos ido haciendo.

Indica los aspectos que menos te han servido y razona porqué

No hay.

La preparación psicológica ¿te ha servido para otros ámbitos ajenos al deporte y la competición? 9

Indica y explica en qué ámbitos te ha servido la preparación psicológica

En los estudios porque cuando llegaban momentos de saturación hemos podido enfocarnos de nuevo en los objetivos y poder abarcarlos todos satisfactoriamente. 
Tabla 6. Valoración de la satisfacción y la eficacia de las técnicas y estrategias utilizadas

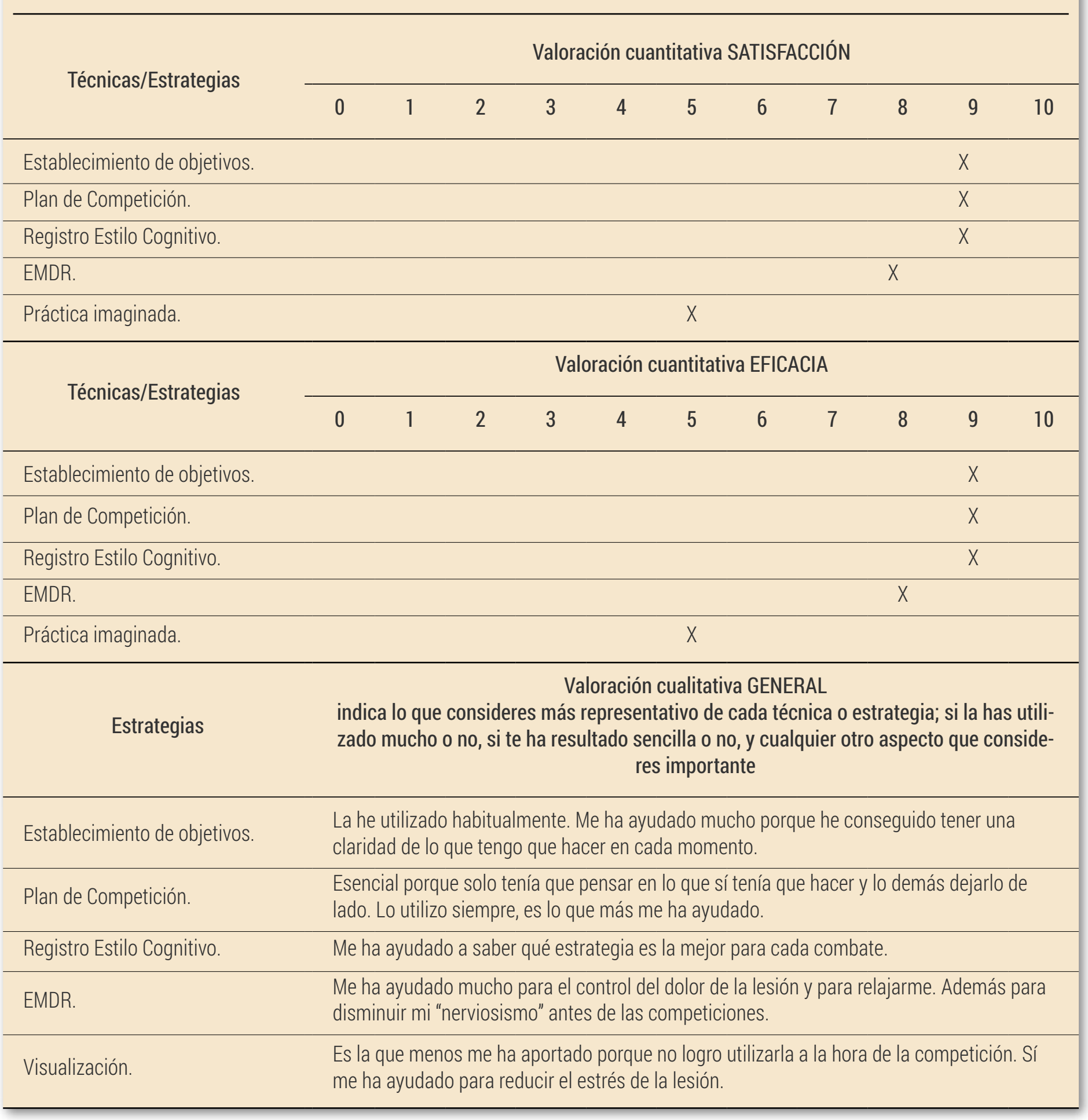

Como se puede observar en la Figura 2, el deportista debía indicar la intensidad del dolor percibido en el día y hasta el momento en que iba a realizar la sesión EMDR (dolor A), para después de la sesión volver a indicar la percepción de dolor justo en ese momento (dolor B).

Finalmente, para la consecución del objetivo 4 se utilizó, entre otros, un registro de adherencia al programa de rehabilitación (Abenza et al., 2011) Ilevado a cabo con el fisioterapeuta (véase Tabla 4).

\section{Valoración de la eficacia de la intervención}

Para valorar el trabajo psicológico realizado se suministró al deportista un cuestionario de valoración que evaluaba, tanto cuantitativa como cualitativamente, la eficacia de la intervención, la satisfacción y la aplicabilidad a otros ámbitos (ver Tabla 5).

En la Tabla 6 se describen las valoraciones cuantitativas y cualitativas que realiza el deportista respecto a algunas de las técnicas psicológicas y estrategias más utilizadas.

\section{Discusión}

Los programas de intervención psicológica sobre la lesión han utilizado habitualmente una combinación de técnicas y estrategias con diferentes objetivos entre los que destacan fundamentalmente el aumento de la adherencia y una mejor recuperación (Brewer, 1998, 2019; Goddard et al., 
2020). El objetivo de este artículo ha sido presentar el trabajo psicológico realizado con un deportista de alto rendimiento de cara a su preparación para los Juegos Paralímpicos de Tokyo 2020. Deportista del CAR de Los Alcázares (Murcia) y sometido a la incertidumbre de realizar su preparación arrastrando una lesión.

Partiendo de los ejes temporal y causal del MGPsLD de Olmedilla y García-Mas (2009) se ubica el trabajo realizado justo después de la lesión (eje temporal), es decir en el proceso de recuperación y/o reinserción, y sobre los consecuentes, es decir los efectos emocionales y psicológicas de la lesión (eje causal). En este contexto es en el que se lleva a cabo la intervención psicológica, actuando sobre las variables psicológicas que se han considerado más relevantes (eje conceptual), básicamente el estrés, los estados de ánimo y los recursos de afrontamiento, todo ello junto al control del dolor.

Dado que esta intervención se ha dirigido, por un lado al rendimiento deportivo, y por otro al proceso de "rehabilitación" de la lesión, el marco de trabajo ha sido considerar las variables psicológicas más relevantes en cada caso, y las técnicas y los recursos de afrontamiento a aplicar y enseñar. En este sentido, la literatura científica ha mostrado que el entrenamiento psicológico es muy importante para la mejora y/o mantenimiento del rendimiento deportivo, produciendo resultados muy positivos en variables como la motivación, concentración o confianza, con técnicas como la práctica imaginada o el establecimiento de objetivos, entre otros (Aoyagi et al., 2017; Brown y Fletcher, 2017), y que como indican Bradberry y Greaves (2012) respecto a la capacidad de entrenamiento de las funciones psicológicas, los neurólogos defienden la plasticidad o capacidad del cerebro para cambiar, por lo que el cerebro puede ser modificado, al igual que un músculo puede desarrollarse mediante el entrenamiento continuado.

Como se indicó, el trabajo para fortalecer la confianza en los combates e incrementar la capacidad de concentración en competición ha sido uno de los pilares. Diversos estudios han puesto de manifiesto que la práctica imaginada puede mejorar el rendimiento de los deportistas en aspectos como la autoconfianza, motivación y control de la ansiedad competitiva (Emara, 2012) sobre todo cuando existen situaciones que el deportista no puede afrontar frecuentemente en la realidad (Pain et al., 2011), como es una lesión deportiva grave. Es por esto, que el profesional de la Psicología del Deporte debe estar cada vez más preparado para intervenir con eficacia adaptándose a las características y demanda de la intervención, adquiriendo y utilizando las competencias profesionales adecuadas a su trabajo (Peris-Delcampo y Cantón, 2018).
Para la evaluación exhaustiva de las variables psicológicas puede ser importante la opinión de los propios deportistas de una forma abierta y subjetiva. La investigación cualitativa permite trabajos de calidad en este sentido, pues trabaja a partir de la información directa de los participantes, buscando en muchas ocasiones responder el por qué y el cómo, algo absolutamente necesario (Molina et al., 2015). En este sentido, la utilización de la técnica EMDR en intervenciones psicológicas, se ha mostrado eficaz para el tratamiento de estrés postraumático (Barron et al., 2019; Marín et al., 2016) así como en los síntomas de ansiedad o dolor. Esto último se persigue con esta intervención: el control del dolor a través de la intervención en EMDR. Tal y como señala el propio deportista (tabla 2) el EMDR "me ha ayudado mucho para el control del dolor... y para relajarme". Pero poco se conoce de su utilización en el tratamiento de lesiones deportivas (Marín et al., 2016) y escasos son los estudios que la usan para tal finalidad. Por otro lado, cuando se evalúa el dolor del deportista, en muchas ocasiones aparecen discrepancias entre lo que valora el profesional y lo que valora aquel que padece el dolor. Es por esto que la mejor forma de evaluar el dolor es preguntar directamente al deportista lesionado, para lo cual es utilizada la Escala Visual Analógica, cuya eficacia está ampliamente demostrada en la evaluación del dolor en deportistas (Gil-Moreno-de Mora et al., 2017; Peñafiel-León, 2018).

Tras la intervención psicológica realizada y la valoración que hace el deportista de la misma (ver Tabla 5), se pueden considerar los siguientes aspectos: a) el deportista está bastante satisfecho con la intervención realizada, destacando el tener los objetivos claros; b) considera que ha sido fundamental para su rendimiento deportivo, destacando una mejor gestión del estrés de la competición; c) considera que le ha ayudado mucho para afrontar la lesión, destacando el control del dolor y la planificación; y d) también considera que le ha ayudado mucho para sus estudios. Respecto a las técnicas y estrategias utilizadas, destacan dos aspectos interesantes; primero, las técnicas clásicas utilizadas en otros estudios (Blumenstein y Orbach, 2020; Didymus y Fletcher, 2017; Konter et al., 2019; Sibley y Bergman, 2018), como el establecimiento de objetivos, el estilo cognitivo y el plan de competición son muy valoradas, tanto a nivel de satisfacción como de eficacia; segundo, destaca el hecho de que la práctica imaginada, una de las técnicas más relevantes en el entrenamiento psicológico (Bompa et al., 2019; Olmedilla et al., 2019; Robin y Fochlay, 2019), no haya sido una de las herramientas preferidas por el deportista; tal y como él mismo expresa "... es la técnica más compleja que he trabajado a nivel psicológico,... no podía sacarle partido en la competición". 
Por último, indicar que un elemento muy importante del trabajo presentado es el hecho de haber podido trabajar con otros profesionales a nivel de rendimiento deportivo (entrenador y coordinador centro), y de salud/lesión (fisioterapeuta, médicos), lo que ha podido ayudar tanto a lo primero como a lo segundo. En el caso de la lesión, el trabajo multidisciplinar es señalado como necesario por diferentes autores (Hess, 2019; Olmedilla y García-Mas, 2009; Wiese-Bjornstal, 2019), y de hecho parece ser cada vez más frecuente. En cualquier caso, a veces la coordinación entre los profesionales no es fácil, y quizá en este trabajo, la extensa carrera deportiva del deportista, las exigencias por la pertenencia a un Centro de Alto Rendimiento, así como la facilidad de acceso para el apoyo y asesoramiento de personal cualificado (médicos, entrenadores, fisioterapeutas, psicólogos, etc.) podría haber supuesto una mejor disposición general hacia el aprendizaje psicológico, máxime cuando su objetivo deportivo consistía en la participación en los JJOO.

En lo que respecta a las limitaciones del estudio, en este caso, al ser un estudio de caso único, presenta limitaciones propias de los estudios de caso (León y Montero, 2015), como son el sesgo del profesional que interviene, la dificultad de controlar todas las variables intervinientes - la dificultad de generalizar las conclusiones al tratarse del análisis de un solo sujeto.

Para finalizar y con el propósito de que este trabajo sirva de guía y ayuda a futuros psicólogos que quieran trabajar en el mundo del deporte, se ha de eliminar la imagen del psicólogo en un despacho y tras un ordenador. Es necesario estar presente en los lugares de entrenamiento y ser "psicólogos de chándal". Hay que adaptar el trabajo a las circunstancias y facilitar que los deportistas y el cuerpo técnico vean al psicólogo como un miembro más del cuerpo técnico, disponibles para ayudarlos ante cualquier dificultad, dentro y fuera del tatami.

\section{Aplicaciones Prácticas}

A pesar de las limitaciones, los estudios de caso son necesarios para difundir la parte práctica de los psicólogos del deporte ofreciendo herramientas útiles a los profesionales aplicados en este campo.

En cuanto a las perspectivas futuras del presente trabajo, es pertinente el seguimiento individual del deportista, no solo teniendo en cuenta las habilidades psicológicas deportivas básicas, sino aspectos extradeportivos como familia, trabajo, relaciones afectivas, etc. Igualmente, sería importante el aumento de trabajos específicos sobre la evaluación de los aspectos que rodean el proceso lesional y la rehabilitación de los deportistas, centrándose dichos estudios en deportes concretos, ya que aunque es lógica la aparición de las variables consideradas como frecuentes o clásicas, sería interesante recoger información de interés para el entrenamiento psicológico especifico en deportes concretos, de manera que se pudiera dotar a deportistas y entrenadores de una correcta formación sobre la definición de las variables psicológicas que permitieran unificar el lenguaje empleado en el entorno deportivo.

Por otro lado, la evaluación de los aspectos psicológicos relacionados con el momento lesional puede servir para crear programas de entrenamiento psicológico de mayor calidad, adaptados a cada deporte y a los momentos concretos de la temporada. La estructura competitiva de cada deporte es distinta, por lo que el trabajo psicológico también lo será.

\section{Referencias}

Abdullah, M. R., Musa, R. M., Maliki, A. B. H. M. B., Kosni, N. A. y Suppiah, P. K. (2016). Role of psychological factors on the performance of elite soccer players. Journal of Physical Education and Sport, 16(1), 170-176. https://doi.org/10.7752/ jpes.2016.01027

Abenza, L., González, J., Reyes, L., Reyes, F., Blas, A. y OlmediIla, A. (2014). Descripción y evaluación del entrenamiento psicológico de una deportista de regata clase laser radial. Revista Iberoamericana de Psicología del Ejercicio y el Deporte, 9(1), 67-92

Abenza, L., Olmedilla, A. y Martinez, C. (2017). Propuesta de entrenamiento psicologico integrado al deportivo en el tenis de mesa: una experiencia en el CAR Sant Cugat de Barcelona. Informacio Psicologica, 112, 74-94. http://dx.medra.org/10.14635/ IPSIC.2016.112.6

Abenza, L., Olmedilla, A. y Ortega, E. (2010). Efectos de las lesiones sobre las variables psicológicas en futbolistas juveniles. Revista Latinoamericana de Psicología, 42(2), 265-277. https://doi.org/10.14349/rpl.v42i2.479

Abenza, L., Olmedilla, A., Ortega, E. y Esparza, F. (2011). Construcción de un registro de conductas de adherencia a la rehabilitación de lesiones deportivas. Revista de Psicología del Deporte, 20(2), 455-476

Abenza, L., Olmedilla, A., Ortega, E., Ato, M. y García-Más, A. (2010). Análisis de la relación entre el estado de ánimo y las conductas de adherencia en deportistas lesionados. Anales de Psicología/ Annals of Psychology, 26(1), 159-168.

Alexander, D., Bloom, G. A. y Taylor, S. L. (2020). Female Paralympic athlete views of effective and ineffective coaching practices. Journal of Applied Sport Psychology, 32(1), 48-63.

Álvarez, O., Estevan, I., Falcó, C., Hernández-Mendo, A. y Castillo, I. (2014). Perfil de habilidades psicológicas en taekwondistas universitarios y su relación con el éxito en competición. 
Cuadernos de Psicología del Deporte, 14(3), 13-20. https://doi. org/10.4321/S1578-84232014000300002

Aoyagi, M. W., Poczwardowski, A., Statler, T., Shapiro, J. L. y Cohen, A. B. (2017). The performance interview guide: recommendations for initial consultations in sport and performance psychology. Professional Psychology: Research and Practice 48(5), 352360. https://doi.org/10.1037/pro0000121.

Aragón, P. O. (2002). Intervención psicológica con deportistas paralímpicos Sydney 2000. Revista de Psicología del Deporte, 17(2), 269-273.

Ashbrook, P. R., Gillham, A. y Barba, D. (2018). Effects of an Individualized Mental-Skills-Training Program on Golf Performance: A Single-Subject Research Design. The Sport Psychologist, 32(4), 275-289.

Barron, I.G., Bourgaize, C., Lempertz, D., Swinden, C. y Darker-Smith, S. (2019). Eye movement desensitization reprocessing for children and adolescents with posttraumatic stress disorder: a systematic narrative review. Journal of EMDR Practice and Research, 13(4). DOI: https://doi.org/10.1891/19333196.13.4.270

Blumenstein, B. y Orbach, I. (2020). Periodization of psychological preparation within the training process. International Journal of Sport and Exercise Psychology, 18(1), 13-23. https://doi.org/10.1 080/1612197X.2018.1478872

Bompa, T., Blumenstein, B., Hoffmann, J., Howell, S. y Orbach, I. (2019). Integrated periodization in sports training \& athletic development: Combining training methodology, sports psychology, and nutrition to optimize performance. Meyer \& Meyer Sport.

Bradberry, T. y Greaves, J. (2012). Inteligencia emocional 2.0. Estrategias para conocer y aumentar su coeficiente. Conecta.

Brewer, B. W. (1998). Adherence to sport injury rehabilitation programs. Journal of Applied Sport Psychology, 10(1), 70-82.

Brewer, B. W. (2019). Adherence issues in the prevention and rehabilitation of sport injuries. In M. H. Anshel, T. A. Petrie, y J. A. Steinfeldt (Eds.), APA Handbooks in Psychology series. APA Handbook of Sport and Exercise Psychology, Vol. 1. Sport Psychology (pp. 727-741). American Psychological Association. https:// doi.org/10.1037/0000123-036

Brown, D. J. y Fletcher, D. (2017). Effects of psychological and psychosocial interventions on sport performance: A meta-analysis. Sports Medicine, 47(1), 77-99. https://doi.org/10.1007/ s40279-016-0552-7

Carazo, P. y Araya, G. (2010). Perfil de rasgos psicológicos para el rendimiento deportivo en hombres y mujeres practicantes de taekwondo. Revista Iberoamericana de Psicología del Ejército y el Deporte, 5, 253-266.

Chica, A.C., Guirval, F.G., Garrido, R.E.R., Chaves, G.C. y Mendo, A.H. (2019). Efectos de un programa de danza española en mujeres con fibromialgia. Cuadernos de Psicología del Deporte, 19(2), 52-69. https://doi.org/10.6018/cpd.343401

Didymus, F. F. y Fletcher, D. (2017). Effects of a cognitive-behavioral intervention on field hockey players' appraisals of organizational stressors. Psychology of Sport and Exercise, 30, 173-185. https://doi.org/10.1016/j.psychsport.2017.03.005

Emara, A. (2012). Strategies of mental imagery and it's relation to some psychological variables in sport of wrestling. World Journal of Sport Sciences, 6, 167-172. https://doi.org/10.5829/idosi. wjss.2012.6.2.1131
García-Naveira, A. (2017). Psychological training for improving self control in a football coach. Acción Psicológica, 14(1), 27-42. https://doi.org/10.5944/ap.14.1.19252

Gil-Moreno-De-Mora, G., Palmi, J. y Prat-Subirana, J. A. (2017). Valoración de la percepción subjetiva de la fatiga en motoristas de competición rally-raid Dakar. Acción Psicológica, 14(1), 93104. https://doi.org/70.5944/ap. 14.7.19265

Goddard, K., Roberts, C. M., Byron-Daniel, J. y Woodford, L. (2020). Psychological factors involved in adherence to sport injury rehabilitation: a systematic review. International Review of Sport and Exercise Psychology, publicación en avance online. https:// doi.org/10.1080/1750984X.2020.1744179

Golby, J. y Wood, P. (2016). The effects of psychological skills training on mental toughness and psychological well-being of student-athletes. Psychology, 7, 901-913. https://doi.org/10.4236/ psych.2016.76092

González-Hernández, J. (2017). Diseño del entrenamiento mental del tenista. De lo científico a lo aplicado. Revista de Psicología Aplicada al Deporte y el Ejercicio Físico, 2(1), Artículo e5. https://doi.org/10.5093/rpadef2017a5

Gross, M., Moore, Z. E., Gardner, F. L., Wolanin, A. T., Pess, R. y Marks, D. R. (2018). An empirical examination comparing the mindfulness-acceptance-commitment approach and psychological skills training for the mental health and sport performance of female student athletes. International Journal of Sport and Exercise Psychology, 16, 431-451.

\section{https://doi.org/10.1080/1612197X.2016.1250802}

Haslett, D., Choi, I. y Smith, B. (2020). Para athlete activism: A qualitative examination of disability activism through Paralympic sport in Ireland. Psychology of Sport and Exercise, 47, 101639.

Hess, C. W., Gnacinski, S. L. y Meyer, B. B. (2019). A Review of the Sport-Injury and-Rehabilitation Literature: From Abstraction to Application. The Sport Psychologist, 33(3), 232-243. https://doi. org/10.1123/tsp. 2018-0043

Kinugasa, T. (2013). The application of single-case research designs to study elite athletes' conditioning: An update. Journal of Applied Sport Psychology, 25(1), 157-166.

Konter, E., Beckmann, J. y Mallett, C. J. (2019). Psychological skills for football players. Football Psychology: From Theory to Practice. Routledge, Taylor \& Francis.

Kristiansen, E., Roberts, G. C. y Abrahamsen, F. E. (2008). Achievement involvement and stress coping in elite wrestling. Scandinavian Journal of Medicine \& Science in Sports, 18(4), 526-538. https://doi.org/10.1111/i.1600-0838.2007.00646.X.

León, O. G. y Montero, I. (2015). Métodos de investigación en Psicología y Educación: las tradiciones cuantitativa y cualitativa, (4º Ed.). Mc Grawhill.

Liberal, R., Escudero, J. T., Cantallops, J y Ponseti, J. (2014). Impacto psicológico de las lesiones deportivas en relación al bienestar psicológico y la ansiedad asociada a deportes de competición. Revista de Psicología del Deporte, 23(2), 451-456.

Lim, T. H., Jang, C. Y., O'Sullivan, D. y Oh, H. (2018). Applications of psychological skills training for Paralympic table tennis athletes. Journal of Exercise Rehabilitation, 14(3), 367-374. https:/l doi.org/10.12965/jer.1836198.099

Lundqvist, C. Stahl, L., Kenttä, G. y Thulin, U. (2018). Evaluation of a mindfulness intervention for Paralympic leaders prior to the Paralympic Games. International Jour- 
nal of Sports Science \& Coaching, 13(1), 62-71. https://doi. org/10.1777/17747954177746495

MacNamara, A., Button, A. y Collins, D. (2010). The role of psychological characteristics in facilitating the pathway to elite performance part 1: Identifying mental skills and behaviors. The Sport Psychologist, 24(1), 52-73. https://doi.org/70.1123/tsp.24.7.52

Mahamud, J., Tuero, C. y Márquez, S. (2005). Características psicológicas relacionadas con el rendimiento: comparación entre los requerimientos de los entrenadores y la percepción de los deportistas. Revista de Psicología del Deporte, 14(2), 237-251.

Marín, C., Guillén, A. I. y Vergara, S. (2016). Nacimiento, desarrollo y evolución de la desensibilización y el reprocesamiento por medio de movimientos oculares (EMDR). Clínica y Salud, 27(3), 101-114. https://doi.org/10.1016/i.clysa.2016.09.001

Martin, J., Vassallo, M., Carrico, J. y Armstrong, E. (2019). Predicting Happiness in Paralympic Swimming Medalists. Adapted Physical Activity Quarterly, 36(3), 309-324.

Martín, V., Blasco, M. J., Casals, M., Fernández-Villa, T., Molina, A. J., Martínez, F. V., Martín, A., Langohr, K. y Ayán, C. (2018). Incidencia de las lesiones en la competición de lucha leonesa y factores asociados (2005-2015). Apunts Medicina de l" Esport, 53(199), 105-112.

McPherson, M. y Pickett, W. (2010). Characteristics of martial art injuries in a defined Canadian population: a descriptive epidemiological study. BioMedCentral Public Health, 10, artículo 795. https://doi. org/70.1786/1471-2458-10-795

Molina, P., Guillamón, M. y Úbeda, J. (2015). La investigación cualitativa en las revistas españolas de ciencias del deporte (20072011). Revista de Psicología del Deporte, 24(1), 29-36.

Moreno-Fernández, I. M., Gómez-Espejo, V., Olmedilla-Caballero, B., Ramos-Pastrana, L. M., Ortega-Toro, E. y Olmedilla, A. (2019). Eficacia de un programa de preparación psicológica en jugadores jóvenes de fútbol. Revista de Psicología Aplicada al Deporte y al Ejercicio Físico, 4(2), Artículo e14, 1-7. https://doi. org/10.5093/rpadef2019a13

Olmedilla, A. y García-Mas, A. (2009). El modelo global psicológico de las lesiones deportivas. Acción Psicológica, 6(2), 77-91.

Olmedilla, A., García-Más, A. y Ortega, E. (2017). Características psicológicas para el rendimiento deportivo en jóvenes jugadores de fútbol, rugby y baloncesto. Acción Psicológica, 14(1), 7-16. https://doi.org/10.5944/ap. 14.19249

Olmedilla, A., Moreno-Fernández, I. M., Gómez-Espejo, V., Robles-Palazón, F. J., Verdú, I. y Ortega, E. (2019). Psychological intervention program to control stress in youth soccer players. Frontiers in Psychology, 10:2260. https://doi.org/10.3389/ fpsyg. 2019.02260

Olmedilla, A., Ortega, E., Andreu, M.D. y Ortin, F.J. (2010). Programa de intervención psicológica en futbolistas: evaluación de habilidades psicológicas mediante el CPRD. Revista de Psicología del Deporte, 19(2), 249-262.

Olmedilla, A., Sánchez-Aldeguer, M.F., Almansa, C. M., Gómez-Espejo, V. y Ortega, E. (2018). Entrenamiento psicológico y mejora de aspectos psicológicos relevantes para el rendimiento deportivo en jugadoras de futbol. Revista de Psicología Aplicada al Deporte y al Ejercicio Físico, 3(1), Artículo e8. https://doi. org/10.5093/rpadef2018a2

Pain, M., Harwood, C. y Anderson, R. (2011). Precompetition imagery and music: the impact on flow and performance in com- petitive soccer. The Sport Psychologist, 25(2), 212-232. https:// doi.org/10.1123/tsp.25.2.212

Palmi, J., Planas, A. y Solé, S. (2018). Intervención mindfulness de rehabilitación de un deportista lesionado: Caso en fútbol profesional. Revista de Psicología del Deporte, 27(1), 115-122.

Pedraza-Ramirez, I. (2019). Generación LoL: Entrenamiento psicológico mediante una propuesta holística con un equipo profesional de e-sports. Revista de Psicología Aplicada al Deporte y el Ejercicio Físico, 4(1), Artículo e2. https://doi.org/10.5093/ rpadef2019a3

Peñafiel-León, M. P. (2018). Aplicación del método POLD en deportistas de alto rendimiento con lumbalgia que asisten a la Federación Ecuatoriana de Remo, en la ciudad de Guayaquil. (Tesis doctoral). Universidad Católica de Santiago de Guayaquil, Ecuador.

Peris-Delcampo, D. y Cantón, E. (2018). El perfil profesional del psicólogo especialista en psicología del deporte en fútbol. Revista de Psicología Aplicada al Deporte y el Ejercicio Físico, 3(1), Artículo e9. https://doi.org/70.5093/rpadef2018a6

Peris-Delcampo, D., Taipe-Nasimba, N., Expósito, V. y Cantón, E. (2019). Psychological Intervention Using Motivational Coaching in Dance Sport: A Single Case Study. Preprints 2019, 2019040241. https://doi.org/10.20944/preprints201904.0241.v1

Puce, L., Marinelli, L., Mori, L., Pallecchi, I. y Trompetto, C. (2017). Protocol for the study of self-perceived psychological and emotional well-being of Young Paralympic athletes. Health and Quality of Life Outcomes, 15, artículo 219. https://doi.org/10.1186/ s12955-017-0798-2

Robin, N. y Flochlay, C. (2017). Imagerie mentale en sport et applications en EPS. EPS: Revue Education Physique et Sport. Editeur EP\&S.

Robles-Rodríguez, A., Abad-Robles, M. T., Robles-Rodríguez, J. y Giménez, F. J. (2020). Factores psicológicos asociados a la formación y al rendimiento en judokas de élite. Journal of Universal Movement and Performance, 1, 27-37. https://doi. org/10.17561/jump.n1.3

Shapiro, F. (1995). EMDR: Basic principles, protocols, and procedures. Guilford.

Shapiro, F. y Forrest, M. S. (2001). EMDR: Eye movement desensitization and reprocessing. Guilford.

Sibley, B. A. y Bergman, S. M. (2018). What keeps athletes in the gym? Goals, psychological needs, and motivation of CrossFit $^{\text {TM }}$ participants. International Journal of Sport and Exercise Psychology, 16(5), 555-574. https://doi.org/10.1080/161219 7X.2017.7280835

Smith, D., Romano-Smith, S., Wright, D. J., Deller-Rust, B. y Wakefield, C. J. (2019). The Effects of Combining PETTLEP Imagery and Action Observation on Bicep Strength: A Single-Case Design. Journal of Applied Sport Psychology, 32(4), 377-391. https://doi.org/10.1080/10413200.2018.1560372

Sotoodeh, M.S., Talebi, R., Hemayattalab, R. y Arabameri, E. (2012). Comparison of selected metal skills between elite and non-elite male and female taekwondo athletes. World Journal of Sport Sciences, 6(1), 32-38. https://doi.org/70.5829/idosi. wjss.2012.6.1.1107

Torralba, M. A., Braz, M. y Rubio, M. J. (2017). Motivos de la práctica deportiva de atletas paralímpicos españoles. Revista de Psicología del Deporte, 26(1), 49-60. 
Turner, M. J., Ewen, D. y Barker, J. B. (2020). An idiographic single-case study examining the use of rational emotive behavior therapy (REBT) with three amateur golfers to alleviate social anxiety. Journal of Applied Sport Psychology, 32(2), 186-204.

Vallarino, V.T., García, C.R. y Zecchini, V.A. (2020). Evaluación e intervención psicológica en jugadoras de hockey sobre hierba femenino. Cuadernos de Psicología del Deporte, 20(1), 62-74. https://doi.org/10.6018/cpd.406651

Weinberg, R.S. y Gould, D. (2018). Foundations of sport and exercise psychology, 7E. Human Kinetics.

Wiese-Bjornstal, D. M. (2019). Psychological predictors and consequences of injuries in sport settings. In M. H. Anshel, T. A. Pe- trie y J. A. Steinfeldt (Eds.), APA handbooks in psychology series. APA handbook of sport and exercise psychology, Sport psychology (Vol. 1, pp. 699-725). American Psychological Association. https://doi.org/10.1037/0000123-035.

Wilkerson, L. A. (1997). Martial arts injuries. The Journal of the American Association, 97(4), 221-226. https://doi.org/10.7556/ jaoa.1997.974.221

Zamora, E. A., Rubio, V. y Hernández, J. M. (2017). Intervención psicológica para el control de la ansiedad en un deportista español de lucha grecorromana. Acción Psicológica, 14(2), 211 224. https://doi.org/10.5944/ap.14.1.15785 